\title{
Muscular Production of Individuated Finger Movements: The Roles of Extrinsic Finger Muscles
}

\author{
Marc H. Schieber \\ Departments of Neurology and Neurosurgery (Neurology), and of Anatomy and Neurobiology, Washington University \\ School of Medicine, Saint Louis, Missouri 63110
}

Individuated finger movements - those in which one or more fingers are moved relatively independently of the movement or posture of other body parts-are produced in part by the action of the extrinsic finger muscles. Flexion/extension movements of the fingers are particularly dependent on these extrinsic muscles, most of which are multitendoned. How can contraction of multitendoned muscles move one digit without producing equivalent motion in other digits?

This question was addressed by recording EMG activity from muscles of the forearm as trained rhesus monkeys performed flexion and extension individuated movements of each digit of the hand and of the wrist. Recordings showed that during movements of different fingers, a given muscle could act as an agonist, antagonist, or stabilizer of the digits it serves. Furthermore, during a given finger movement, several different muscles typically were active.

A three-level connection model was developed that computed the relative motion of the digits during each finger movement based on the changes in EMG activity in the recorded muscles. The model showed that EMG activity changes in the extrinsic finger muscles, and the thenar muscles, could account for most of the motion of both the instructed digit and noninstructed digits. These results indicate that individuated finger movements were produced not by independent sets of muscles acting on each digit, but by the activity of several muscles, many of which act on more than one digit, combined such that the net effect was movement of one digit more than others.

[Key words: electromyography, finger, model, monkey, movement, multitendoned, muscle, rhesus I

Individuated finger movements often are assumed to be produced by the contractions of independent muscles moving each digit, but the anatomy of the extrinsic finger muscles shows otherwise. Primate fingers are flexed and extended mainly by extrinsic muscles that send tendons to two or more digits. For example, the macaque flexor digitorum profundus sends tendons to all five digits (Howell and Straus, 1933; Serlin and Schieber, 1993). Even in humans, flexor digitorum profundus,

Received Nov. 19, 1993; revised May 27, 1994; accepted June 16, 1994.

I thank M. Hayles for editorial assistance and B. Forgash for help in preparing the manuscript. This work was supported by Grant R01-NS27686 from the National Institute of Neurologic Disorders and Stroke.

Correspondence should be addressed to Marc H. Schieber, Washington University School of Medicine, Department of Neurology, 660 South Euclid Avenue, Box 8111 , St. Louis, MO 63110.

Copyright (c) 1995 Society for Neuroscience $0270-6474 / 95 / 150284-14 \$ 05.00 / 0$ flexor digitorum superficialis, and extensor digitorum communis each send tendons to all four fingers (Fahrer, 1981). How can contraction of such muscles move one digit without equivalent motion in other digits?

One possible solution to this paradox might lie in neuromuscular compartmentalization (English, 1984; English and Weeks, 1987; Loeb, 1989; Chanaud et al., 1991). If the tendon to each digit were served by a separate neuromuscular compartment, then a multitendoned muscle could act as a number of separate muscles; and functionally, each finger would have its own independent set of muscles. Although some multitendoned extrinsic finger muscles show some evidence of compartmentalization, no multitendoned muscle has been shown to have functionally separate compartments for each digit it serves (Schieber, 1993a; Serlin and Schieber, 1993; S. C. Gandevia, personal communication). Though availablc cxpcrimental evidence does not exclude neuromuscular compartmentalization in these muscles, neither does it provide an adequate explanation of individuated finger movements.

A second possible solution might be that finger movements are individuated by action of the intrinsic hand muscles, each of which does serve just one digit. Electromyographic studies in humans have suggested, however, that the intrinsic muscles are primarily responsible for controlling the relative degree of flexion and extension at the metacarpophalangeal, proximal interphalangeal, and distal interphalangeal joints within a finger (Long and Brown, 1964; Landsmeer and Long, 1965; Long, 1968; Close and Kidd, 1969; Brandell, 1970; Long et al., 1970; Basmajian, 1978). Though the intrinsic muscles may contribute to individuation of overall flexion and extension of the fingers, they are unlikely to serve a primary role here.

A third possible solution might be that finger movements are individuated by the combined action of more than one extrinsic multitendoned muscle. If each multitendoned muscle produced a different distribution of tension on the digits it serves, then various combinations of activity in these muscles would produce various degrees of movement in different digits. Moreover, while certain muscles move one digit, other muscles might stabilize other digits. In this way, movement of one digit more than others could be produced as the net effect of tension applied simultaneously by several muscles to many digits.

To determine how muscles produce individuated finger movements, EMG activity was recorded from forearm muscles as trained rhesus monkeys performed flexion and extension movements of each digit of the right hand and of the wrist.

This work was previously presented in abstract form (Schieber, 1993b). 


\section{Materials and Methods}

Behavioral task. Three juvenile rhesus monkeys (Macaca mulatta; $\mathrm{K}$, a $6 \mathrm{~kg}$ male; $\mathrm{S}$, a $4 \mathrm{~kg}$ male; and $\mathrm{H}$, a $4 \mathrm{~kg}$ female) were trained to perform visually cued individuated finger movements. The behavioral paradigm and the finger movements made by monkeys $K$ and $S$ have been described in detail elsewhere (Schieber, 1991). In brief, the monkey's elbow was restrained in a molded cast and the right hand was placed in a pistol-grip manipulandum that separated each finger into a different slot. At the end of each slot, each fingertip lay between two microswitches. By flexing or extending a digit a few millimeters, the monkey closed the ventral or dorsal switch, respectively. The manipulandum, in turn, was mounted on an axis permitting flexion and extension wrist movement. The monkey viewed a display on which each digit (and the wrist) was represented by a row of five light-emitting diodes (LEDs). When the monkey flexed or extended a digit, closing a microswitch, the central yellow LED went out and a green LED to the left or right, respectively, came on. Red LEDs to the far left or right were illuminated one at a time, under microprocessor control, instructing the monkey to close that one switch (or move the wrist). If the monkey closed the instructed switch within the $700 \mathrm{msec}$ allowed after illumination of the red instruction LED, and held it closed for a 500 msec final hold period without closing any other switches, he or she received a water reward. After each rewarded trial, the finger movement to be instructed for the next trial was rotated in a pseudorandom order. Unlike monkeys $\mathrm{K}$ and $\mathrm{S}$, monkey $\mathrm{H}$ was initially trained with the wrist axis constantly locked, eliminating the need for active control of wrist position (Schieber, 1993a). After initial EMG recordings, monkey $\mathbf{H}$ was retrained with the wrist axis unlocked. Unless otherwise indicated, all EMGs from monkey $\mathbf{H}$ considered in this report were obtained after retraining with the wrist moving.

Examination of the finger movements generated by these monkeys showed that in each rewarded trial, the digit the monkey had been instructed to move underwent more movement than any other digit (Schieber, 1991). Moreover, each digit had its greatest excursion when it was the instructed digit. In some movements, particularly when the monkey was instructed to flex the thumb or wrist, other digits remained stationary. In other movements, however, noninstructed digits moved to a greater or lesser degree. Each movement is therefore referred to as an instructed movement of a given digit in a given direction, recognizing that there was often some movement of noninstructed digits. For brevity, each instructed movement is denoted by the number of the instructed digit ( 1 for the thumb through 5 for the little finger, $W$ for the wrist), and the first letter of the instructed direction (f for flexion, e for extension); thus, " $2 \mathrm{f}$ " denotes instructed flexion of the index finger.

Recording and stimulation. Once a monkey performed the finger movement task correctly on 70-90\% of the trials, EMGs were recorded from muscles of the right forelimb with techniques described in detail elsewhere (Schieber, 1993a). Since each of these monkeys subsequently underwent studies of single neuron activity in cortical motor areas, surgical implantation of EMG elcctrodes was considered undesirable. Therefore, electrode tips were placed percutaneously in target muscles based on surface landmarks and muscular anatomy (Howell and Straus, 1933; Berringer et al., 1968; Szebenyi, 1969; Fetz and Cheney, 1980; Schieber, 1993a; Serlin and Schieber, 1993). While the investigator held the monkey's hand or forearm, bipolar twisted-pair fine-wire electrodes (impedance of $0.3-1.0 \mathrm{M} \Omega$ at $1 \mathrm{kHz}$ ) were placed percutaneously with 23 or 25 gauge hypodermic needles in different muscles. Although the monkey experienced the transient discomfort of the needle sticks, the needle was withdrawn after each each electrode placement, leaving only the fine-wire electrodes in place. Thereafter the monkey appeared not to experience discomfort, performing the finger movement task with the same proficiency observed in training sessions without EMG electrodes (cf. Basmajian, 1978; Lemon et al., 1990). Electrode insertion points in monkcys $\mathrm{K}$ and $\mathrm{H}$ were recorded on a standardized map of the forearm. Signals from the electrodes were amplified $10,000 \times$, filtered $(300 \mathrm{~Hz}$ to $3 \mathrm{kHz})$, full-wave rectified, integrated, and converted to a pulse frequency replica. Pulse frequency converted EMGs were collected from up to three muscles simultaneously, along with (1) digitized analog signals representing the position of each finger and of the wrist, and (2) event time codes.

Recordings were made from the following muscles: thenar; flexor digitorum profundus, radial region (FDPr); flexor digitorum profundus, ulnar region (FDPu); flexor digitorum superficialis (FDS); flexor carpi radialis (FCR); palmaris longus (PL); flexor carpi ulnaris (FCU); exten-

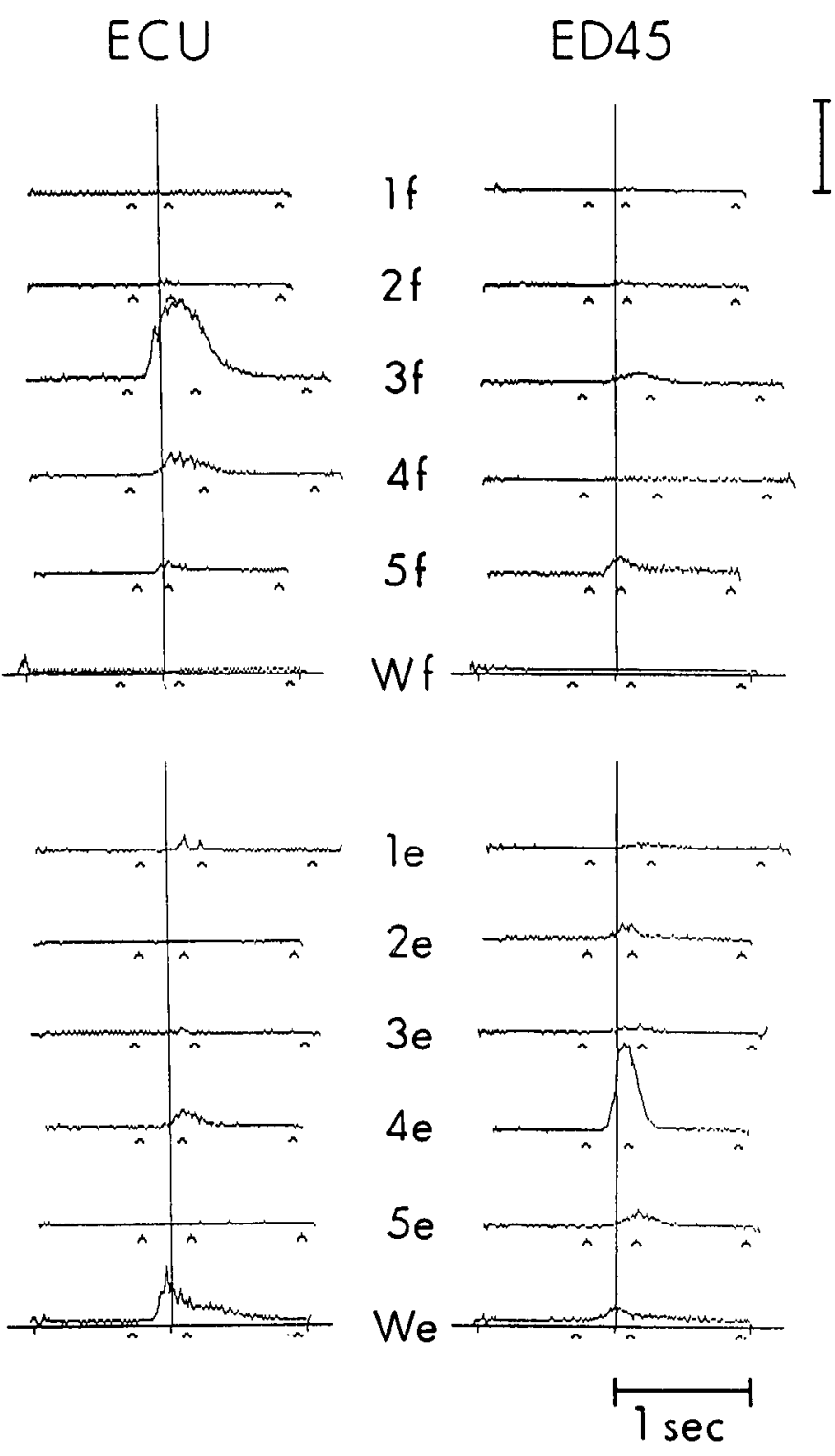

Figure 1. Simultaneous EMG recordings from ECU and ED45. For each muscle, a column shows data as a stack of 12 histograms of activity during the 12 different instructed movements, the six flexions above ( $I f-W f$ ) and the six extensions below (le-We). Although bipolar electrodes in the two muscles were only $10 \mathrm{~mm}$ apart, the ECU electrode recorded intense activity during $3 f$, when little activity appeared in the ED45 electrode, and conversely, the ED45 electrode recorded intense activity during $4 \mathrm{e}$, when little activity appeared in the ECU electrode. Each histogram was compiled in $10 \mathrm{msec}$ bins from the pulse frequency representation of integrated EMG activity recorded during 8-13 correctly performed triais of that instructed movement, with data aligned at the onset of movement in the instructed digit, and was smoothed with a $20 \mathrm{msec}$ moving window. Three carat marks beneath each histogram indicate the average times at which (1) the red instruction/trigger LED was illuminated, (2) the instructed digit's switch closed, and (3) the reward was delivered, in the trials used to compile that histogram. Vertical calibration (arbitrary integrated EMG units): $E C U, 1000 ; E D 45$, 750 .

sor pollicis longus (EPL); extensor digiti secundi et tertii (ED23); extensor digitorum communis (EDC); extensor digiti quarti et quinti (ED45); extensor carpi radialis longus (ECRL); extensor carpi radialis brevis (ECRB); and extensor carpi ulnaris (ECU). The present study thus required that EMG activity be recorded differentially from small, closely packed muscles, and potentially from distinct compartments within a given muscle. In pilot studies, activity from adjacent muscles could not be resolved using traditional bipolar electrode tip separations of 3-10 
Table 1. Numbers of EMG recordings, intramuscular stimulations, and single motor units

\begin{tabular}{lllll} 
& Monkey & & \\
\cline { 2 - 4 } & $\mathrm{K}$ & $\mathrm{S}$ & S & \\
Flexors & & & & \\
Thenar & $6(1: 4: 0: 1)[7]$ & $2(0: 0: 0: 2)[0]$ & $3(3: 0: 0: 0)[1]$ & $11(4: 4: 0: 3)[8]$ \\
FDPr & $7(3: 2: 1: 1)[9]$ & $2(0: 0: 0: 2)[0]$ & $5(2: 3: 0: 0:)[2]$ & $14(5: 5: 1: 3)[11]$ \\
FDS & $4(0: 4: 0: 0)[4]$ & $1(0: 0: 0: 1)[0]$ & $5(2: 3: 0: 0)[1]$ & $10(2: 7: 0: 1)[5]$ \\
FDPu & $6(4: 1: 0: 1)[9]$ & $5(0: 0: 0: 5)[0]$ & $3(0: 2: 1: 0)[1]$ & $14(4: 3: 1: 6)[10]$ \\
FCR & $6(1: 1: 2: 2)[2]$ & $4(0: 0: 0: 4)[0]$ & $3(1: 2: 0: 0)[1]$ & $13(2: 3: 2: 6)[3]$ \\
PL & $3(2: 1: 0: 0)[2]$ & $1(0: 0: 0: 1)[0]$ & $3(0: 2: 1: 0)[1]$ & $7(2: 3: 1: 1)[3]$ \\
FCU & $4(3: 0: 0: 1)[4]$ & $1(0: 0: 0: 1)[0]$ & $3(0: 2: 1: 0)[2]$ & $8(3: 2: 1: 2)[6]$ \\
Extensors & & & & \\
EPL & $1(0: 0: 0: 1)[1]$ & no data & $1(0: 0: 1: 0)[2]$ & $2(0: 0: 1: 1)[3]$ \\
ED23 & $3(0: 1: 1: 1)[4]$ & no data & no data & $3(0: 1: 1: 1)[4]$ \\
EDC & $8(4: 1: 2: 1)[9]$ & $3(0: 0: 0: 3)[0]$ & $7(4: 2: 1: 0)[6]$ & $18(8: 3: 3: 4)[15]$ \\
ED45 & $5(1: 3: 0: 1)[6]$ & $3(0: 0: 0: 3)[0]$ & $3(2: 1: 0: 0)[2]$ & $11(3: 4: 0: 4)[8]$ \\
ECRL & $4(2: 2: 0: 0)[2]$ & $1(0: 0: 0: 1)[0]$ & $2(0: 2: 0: 0)[0]$ & $7(2: 4: 0: 1)[2]$ \\
ECRB & $2(1: 1: 0: 0)[0]$ & $4(0: 0: 0: 4)[0]$ & $3(2: 1: 0: 0)[1]$ & $9(3: 2: 0: 4)[1]$ \\
ECU & $10(0: 4: 3: 3)[7]$ & $2(0: 0: 0: 2)[0]$ & $5(0: 4: 1: 0)[2]$ & $17(0: 8: 4: 5)[9]$ \\
Subtotal & $69(22: 25: 9: 13)[66]$ & $29(0: 0: 0: 29)[0]$ & $46(16: 24: 6: 0)[22]$ & $144(38: 49: 15: 42)$ \\
\hline
\end{tabular}

Numbers in parentheses indicate the number of recordings where intramuscular stimulation (1) evoked a selective, appropriate contraction at current thresholds from 50 to $500 \mu \mathrm{A},(2)$ evoked a selective, appropriate contraction at current thresholds from 550 to $6000 \mu \mathrm{A}$, (3) failed to evoke a contraction with the currents used, or (4) was not performed. Intramuscular stimulation was not available in studies of monkey $S$. Numbers in brackets indicate the number of single motor units recorded from each muscle in each monkey.

mm (cf. Basmajian, 1978; Schieber and Thach, 1985; Loeb and Gans, 1986). Therefore, electrodes with small bipolar tip separations werc employed $(0.15 \mathrm{~mm}$; Schieber, 1993a; cf. Lemon et al., 1990). In recordings made with these electrodes, single motor unit potentials could be identified during periods of low to moderate EMG activity (cf. Fig. 1 of Schieber, 1993a), though full interference patterns were recorded during intense activity. Although the narrow tip spacing of the electrodes may have focused sampling in a given recording on a subset of a muscle's motor units, a reduced sample should still reflect the activity patterns of a homogeneous motor unit pool. Furthermore, since a number of these muscles potentially consist of more than one functional subdivision (Schieber, 1993a; Serlin and Schieber, 1993), recording a local sample of motor units increased the likelihood of detecting differential activity in such subdivisions.

With these electrodes, different patterns of EMG activity in adjacent muscles could be resolved. For example, Figure 1 shows EMGs recorded in one session simultaneously from two adjacent muscles, ECU and ED 45. The electrodes in these two muscles were inserted approximately $10 \mathrm{~mm}$ apart. The electrode in ECU recorded intense activity during movement $3 \mathrm{f}$, when the electrode in adjacent ED45 recorded little activity, and conversely, the electrode in ED45 recorded intense activity during movement $4 \mathrm{e}$, when the electrode in ECU recorded little. These electrodes were also able to record distinct patterns of activity from adjacent regions of FDP (Schieber, 1993a). In most instances, then, EMG pickup from adjacent muscles was so small as to have little quantitative effect on the results described below. Although most electrode placements obtained recordings that could be attributed to a single muscle (see below), occasionally a recording showed a pattern of activity that, based on the patterns in most recordings, appeared to be a mixture of activity from two muscles. Such recordings, which presumably resulted from placement of the electrode's tip near the border between two muscles, were excluded from the present analysis.

Because electrodes were inserled percutaneously, the intramuscular position of the recording tips could not be visualized directly. Therefore, recordings were included in the present analysis if in two or more sessions appropriately positioned electrodes obtained similar EMG patterns during finger movements. Table 1 lists the number of recordings from each muscle included from each monkey. In some instances, recordings were included if they were the only recording obtained in a given monkey from a particular muscle, but showed activity patterns consistent with the same muscle in another monkey. The data set available from monkey $S$ includes a number of single recordings, which should be noted in interpreting the data. To verify that patterns of EMG activity were attributed to the correct muscle, in some recordings from monkeys $\mathrm{K}$ and $\mathrm{H}$, intramuscular electrical stimulation was used to confirm the site of electrode placement, and single motor unit recordings were used to confirm the local origin of EMG activity.

For many recordings from monkeys $\mathrm{K}$ and $\mathrm{H}$, supportive evidence that the electrode tip lay in the target muscle was obtained when intramuscular stimulation evoked finger or wrist movements appropriate for the muscle (but see Loeb and Gans, 1986). Intramuscular electrical stimuli were delivered through the recording electrodes via a stimulus isolator (BAK BSI-1), as trains of 12 monopolar or bipolar, $0.2 \mathrm{msec}$, constant current $(50-6000 \mu \mathrm{A})$ pulses at $100 \mathrm{~Hz}$. Stimulation was performed either immediately after electrode placement, as the examiner held the monkcy's arm and hand, or clse after EMGs were recorded, while the monkey's hand remained in the manipulandum. Stimulation confirmed electrode placement in a given muscle if it evoked (1) visible or palpable contraction of the muscle belly, (2) palpable tightening of the muscle's tendon, and/or (3) appropriate movement of the wrist or digits, (4) without similar evidence of contraction in other muscles. If contractions evoked by intramuscular stimulation were inappropriate for the target muscle, then that EMG recording was excluded from the present analysis.

Low-threshold intramuscular stimulation $(50-500 \mu \mathrm{A})$ may evoke observable contractions by stimulating the muscle nerve near the muscle's innervation point (Fetz and Cheney, 1980; Loeb and Gans, 1986). Because the narrow bipolar tip spacing of the present electrodes effectively reduced the tissue volume stimulated by a given current, when the electrode tips were not close to the muscle's inncrvation point, higher currents $(550-6000 \mu \mathrm{A})$ may have been needed to evoke observable movement, or the highest currents employed here may have been ineffective. Excluding recordings from such electrode placements would have excluded recordings from locations in the muscle situated at a distance from the innervation point, where other functional subdivisions might exist (Serlin and Schieber, 1993). Table 1 therefore lists in parentheses the number of recordings where intramuscular stimulation (1) evoked a selective, appropriate contraction at current thresholds from 50 to $500 \mu \mathrm{A}$, (2) evoked a selective, appropriate contraction at current thresholds from 550 to $6000 \mu \mathrm{A}$, (3) failed to evoke a contraction 
A
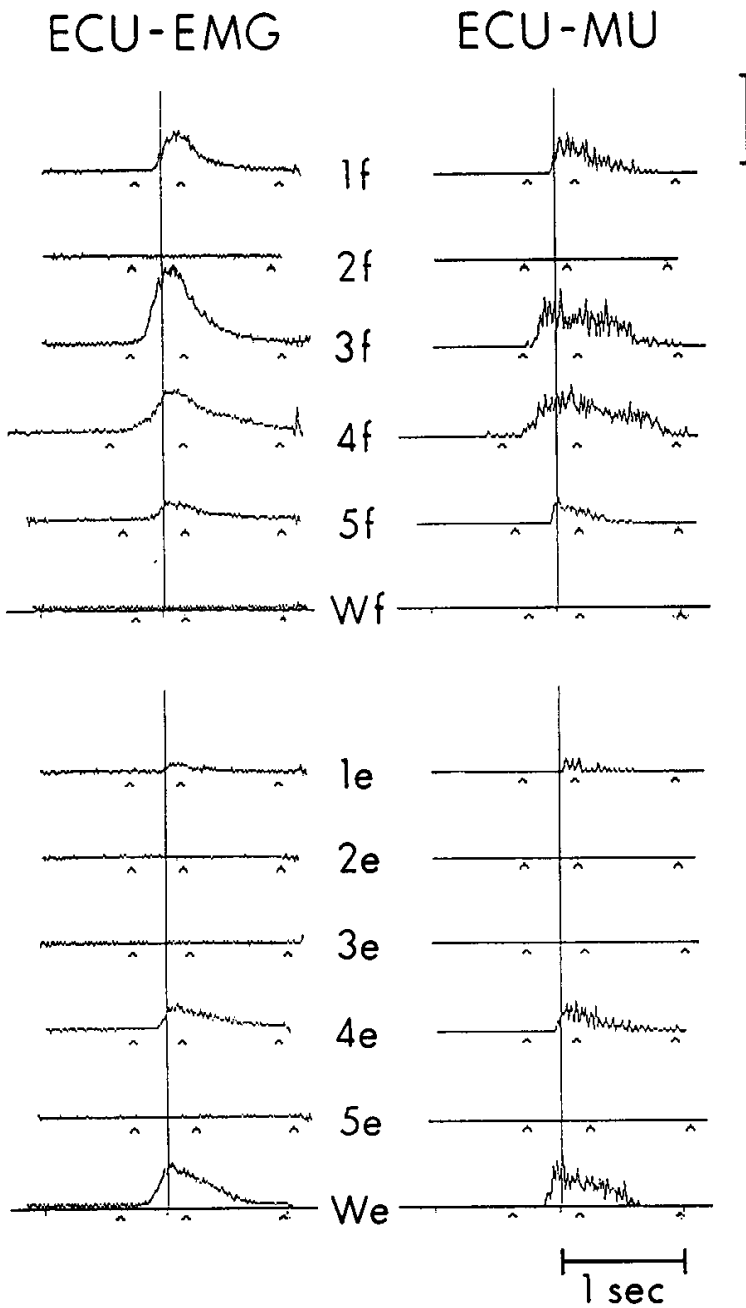

B
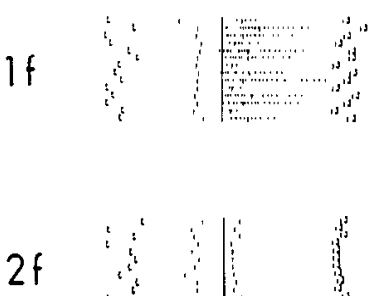

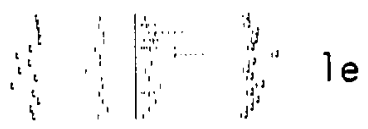

\begin{tabular}{ll|lll}
$r$ & & & \\
3 & & & & \\
3 & & &
\end{tabular}

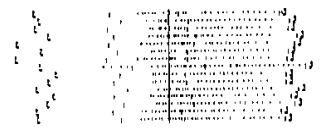

$4 f$
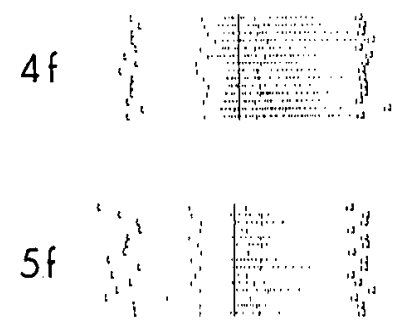

Wf

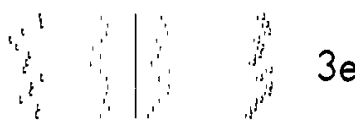

$3 e$

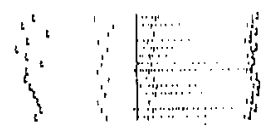

$4 e$

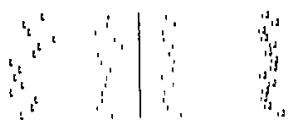

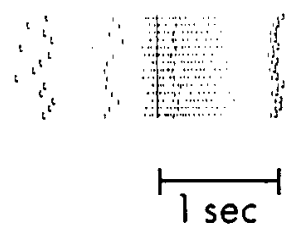

Figure 2. Parent EMG and a single motor unit from ECU. A, The left column shows histograms of EMG activity recorded from ECU during the 12 instructed movements. The right column shows histograms of the discharge of a single motor unit $(M U)$ discriminated from the same recording. Although EMG activity was recorded during several instructed movements for which activity in ECU might not have been expected, the single MU was active during all these movements, indicating that EMG activity during all these different movements arosc from a single motoneuron pool. Conventions for histograms are as for Figure 1. Vertical calibration: ECU-EMG, 750 arbitrary integrated EMG units; $M U, 100 /$ sec. $B$, Rasters of MU discharge during repeated trials of each instructed movement. The histograms in $A$, right, were compiled from these rasters. For each row in the raster, square brackets at left and right mark the beginning and end of data collection for that trial; three ticks mark the times at which the instruction/trigger LED was illuminated, the instructed digit's switch closed, and the reward was delivered; dots mark the times of MU potentials. Rows are aligned on the onset of motion in the instructed digit (vertical line).

with the currents used, or (4) was not performed, all of which were included as recordings from the same muscle since they showed similar patterns of EMG activity during finger movements.

Accurate placement of electrode tips in a given muscle, even when confirmed by electrical stimulation, does not guarantee that all the recorded EMG activity originated in the impaled muscle. Electrodes positioned in one muscle can pick up EMG activity that originates in a neighboring muscle (Loeb and Gans, 1986; English and Weeks, 1989). To minimize the likelihood of recording EMG activity from neighboring muscles, the present study employcd (1) clectrodes with a close tip spacing, and (2) a relatively high low-frequency cutoff filter $(300 \mathrm{~Hz})$. When these filtered EMG signals were audiomonitored with headphones, distant activity could typically be distinguished from local activity by its greater content of low-frequency components, and lesser content of high-frequency components. Recordings contaminated by such distant pickup were excluded from the present analysis.

In addition, recordings of single motor units (MUs) obtained from some electrode placements confirmed that the pattern of parent EMG activity resulted from local MUs. Single MUs are confined to a single muscle, or to a single compartment within a muscle (English, 1984;
Loeb and Gans, 1986; English and Weeks, 1987, 1989; Armstrong et al., 1988; Hammond et al., 1989; but see Emonet-Dénand et al., 1971). A single $\mathrm{MU}$ potential occasionally could be discriminated from parent EMG activity using a dual time/amplitude window (BAK DDIS-1). In such instances, the discriminator's output pulses representing the MU potentials were collected in a channel separate from that of the parent EMG.

Figure 2 illustrates how MU recordings confirmed that EMG activity during different finger movements originated from the same muscle. Histograms of EMG activity recorded in one session from ECU during each of the 12 instructed finger movements are shown on the left in Figure $2 A$. As expected, ECU was active during wrist extension (We), but EMG activity was also recorded during instructed extension of digil 4 or digit 1 ( $4 \mathrm{e}$ or $1 \mathrm{e}$ ), and this activity might have been picked up from the adjacent muscles ED45 or EPL, respectively. Similarly, EMG activity recorded during finger flexions ( $\mathrm{f} f, 3 \mathrm{f}, 4 \mathrm{f}$, and $5 \mathrm{f}$ ) might have been picked up from the adjacent FDPu. A single MU discriminated from the parent EMG was active during all of these movements (Fig. $2 A$, right; $B$ ), however, indicating that at least some of the parent EMG activity recorded during all the different movements arose from the 
same motoneuron pool. Similar examples of single MUs from FDPr and FDPu have been presented elsewhere (Schieber, 1993a).

Not all single MUs showed discharge changes during all the finger movements for which the parent EMG activity changed. Small MUs were sometimes tonically active, presumably at maximal discharge rate, while the parent EMG showed a burst of activity. Large MUs were sometimes inactive during movements involving less intense parent EMG activity, presumably reflecting higher thresholds for these MUs. Allowing for discharge rate saturation and threshold variations, the number of MUs whose activity patterns provided confirmation that parent EMG activity arose locally from each muscle is given in brackets in Table 1.

Data analysis. For each EMG recording, data were collected from 4 15 correctly performed trials of each instructed movement. Off line, each recording was analyzed for changes in EMG activity consistently related to each instructed movement, as described previously (Schieber, 1993a). For each correctly performed trial, a Kolmogorov-Smirnov test detected significant differences $(P<0.05)$ between a control period $(500$ msec preceding illumination of the red instruction LED) and each of three test periods: (1) the premovement period, from $50 \mathrm{msec}$ after illumination of the instruction LED to the onset of movement in the instructed digit; (2) the movement period, from onset of movement to switch closure; and (3) the overall reaction period, from $50 \mathrm{msec}$ after illumination of the instruction LED to switch closure. EMG activity in a recording was considered to change consistently in relation to an instructed movement if a significant change was identified in $90 \%$ or more of the correctly performed trials in any one test period. If a consistent change was identified, the amplitude of the change for that movement was calculated by subtracting the pulse frequency during the 500 msec control period (averaged across trials) from the peak (or trough) of activity during the overall reaction period (measured from a moving average histogram of data from all correctly performed trials). Since EMG activity generally rose from baseline to its peak (or fell to its trough) in an approximately linear trajectory lasting about $100 \mathrm{msec}$, the peak change was roughly proportional to the area under the curve. If no consistent relationship was found, the activity change for that instructed movement was assigned a value of zero.

\section{Results}

The present report is based on 166 EMG recordings made from 14 muscles in three monkeys. Of these, 144 recordings were accepted for the present analysis. Table 1 lists the number of EMG recordings included from each muscle in each monkey. Recordings attributed to EPL or ED23 were not obtained in monkey S, and recordings from ED23 were not obtained in monkey $\mathrm{H}$ after retraining with the wrist moving. Whether these muscles were not impaled despite repeated attempts, or whether they were inadvertently denervated, is uncertain. For clarity of exposition, the present report will describe the results typical of all three monkeys using data obtained in monkey $\mathrm{K}$ (from which the most data was available), noting significant differences among subjects.

Certain of the 14 "muscles" do not conform to the usual definition of a muscle. Thenar muscles were included here to account for thumb flexion, during which FDP, the only extrinsic flexor muscle with a tendon to the thumb, was inactive (see below). Though intramuscular stimulation in the thenar eminence often elicited abduction, flexion, opposition, or adduction of the thumb, all recordings from thenar muscles showed similar patterns of activity during task performance. Whether all these muscles cocontracted, or whether the activity of one or two thenar muscles was recorded throughout the thenar eminence, could not be distinguished (Loeb and Gans, 1986), and therefore data from all thenar recordings was pooled for the present study. Conversely, flexor digitorum profundus in macaques has two functional subdivisions that correspond to two different morphologic regions (Schieber, 1993a; Serlin and Schieber, 1993): the radial subdivision, FDPr, acts on digits 1, 2, and 3; the ulnar subdivision, FDPu, acts on digits 3, 4, and 5. Therefore, FDPr and FDPu are treated here as functionally separate muscles.

Convincing evidence of functional subdivisions in the other multitendoned finger muscles-FDS, ED23, EDC, and ED45was not obtained in the present study, despite deliberate searches. For each of these muscles, recordings obtained from widespread locations showed similar patterns of EMG activity. Because placing multiple clcctrodes in a single muscle appeared to impair the monkeys' performance, most of the data available to compare EMG activity recorded from various locations in a given muscle came from recordings obtained in separate sessions. But in a few sessions recording from monkey $\mathrm{H}$, two or three electrodes were placed concurrently at different locations within a single muscle. Figure 3 shows EMGs recorded simultaneously in one such session from three electrodes placed at separate locations within EDC, selected to record from different morphologic regions of the muscle (cf. Serlin and Schieber, 1993, their Fig. 1). One electrode was placed in EDC proximally; intramuscular stimulation here at $100 \mu \mathrm{A}$ evoked a contraction that extended digits 3 and 4 more than 2 or 5 . A second electrode was placed distally, on the radial side of EDC; stimulation here at $200 \mu \mathrm{A}$ evoked extension of digits 2 and 3 more than 4 or 5 . A third electrode was placed on the ulnar side of EDC, at the same proximodistal level as the second electrode; stimulation here evoked a flexion reflex at currents below threshold for contraction of EDC. The insertion points of the second and third electrodes were $9 \mathrm{~mm}$ apart.

Though distinct effects were obtained by stimulating through the three electrodes, the patterns of EMG activity recorded were remarkably similar. All three showed phasic EMG activity during movements $2 \mathrm{f}, 5 \mathrm{f}, 2 \mathrm{e}, 3 \mathrm{e}$, and We. All three showed EMG activity preceding the instruction signal for movement $3 \mathrm{e}$. Although small quantitative differences among the three recordings can be found, the only qualitative differences were observed during $4 \mathrm{e}$ and $5 \mathrm{e}$. During $4 \mathrm{e}$, phasic activity was recorded from the proximal and ulnar electrodes but not from the radial electrode. During $5 \mathrm{e}$, tonic activity recorded from the proximal and ulnar electrode was not recorded from the radial electrode. These partial differences in simultaneous recordings from different regions of EDC contrast with the overt differences in EMG activity between FPDr and FDPu, which were consistently apparent in recordings obtained from the two regions, even in separate sessions (Schieber, 1993a; see also Fig. 4). Results similar to those shown in Figure 3 were obtained in four double electrode recordings from EDC, two double electrode recordings from FDS, and one triple electrode recording from ED45. (All of these except one double electrode recording from EDC were obtained from monkey $\mathrm{H}$ while she was trained with the wrist axis locked, and were not otherwise included in the analysis below.) Therefore, although some functional subdivisions might exist in FDS, ED23, EDC, or ED45 (Serlin and Schieber, 1993), each of these multitendoned extrinsic finger muscles was considered here as a single functional entity.

\section{Agonist, antagonist, and stabilizing activity}

Figure 4 shows a typical EMG recording from each muscle (flexors above, extensors below) during each of the 12 instructed movements. Each trace is a moving average histogram of activity recorded during 8-12 successfully performed trials of an instructed movement. Examination of the column for each muscle shows that, although thenar muscles were active only during If, and ECRL was active only during $5 \mathrm{e}$, most muscles were 
active during more than one instructed movement. Conversely, examination of the row for each instructed movement shows that, although if involved only the thenar muscles, most movements involved EMG activity in more than one muscle.

Consideration of each muscle's mechanical connections indicated that many muscles were active as agonists or antagonists of the instructed digit's motion. FDPr, for example, has tendinous connections that cross the wrist and attach to digits 1 , 2 , and 3 . FDPr was active during $2 \mathrm{f}, 3 \mathrm{f}$, and $\mathrm{Wf}$, when it could have contributed to flexion of digit 2 , of digit 3 , or of the wrist, respectively. FDPr was also active during $2 \mathrm{e}$, when it could have antagonized extension of digit 2. Many muscles were also active during movements in which they could have no effect on the instructed digit. Consideration of the muscle's mechanical connections in these instances often suggested that their activity stabilized noninstructed digits. For example, FDPr was active during $5 \mathrm{e}$, when it could not have served either as an agonist or antagonist. Tension in FDPr during 5e might, however, have limited extension of digits 2 and 3 that otherwise would have resulted from the contraction of EDC used to extend digit 5. In different instructed movements, then, FDPr acted as an agonist, antagonist, or stabilizer.

To evaluate further the patterns of EMG activity in each muscle, thc following analysis was performed for each monkey. For each EMG recording, the change in activity from the premovement baseline to the peak (or trough) activity during movement was computed for each instructed movement (see Materials and Methods). These activity changes for each recording were normalized such that the largest change was assigned a value of $100 \%$, thereby minimizing differences among recordings that resulted from variability in positioning, orientation, and impedance of electrode tips (Loeb and Gans, 1986). The mean and standard deviation of the changes for each movement then were computed across recordings from a given muscle in a given monkey. These averaged, normalized changes during each movement for each muscle in monkey $\mathrm{K}$ are shown in Figure 5.

In this figure, the normalized activity changes for each muscle during the 12 instructed movements are plotted in a row. Flexor muscles are shown in the top seven rows; extensor muscles, in the bottom seven rows. Different symbols distinguish the rows of data from different muscles. Instructed flexions (left) and instructed extensions (right) have been separated. Potential agonists are the flexor muscles during instructed flexions, the extensor muscles during instructed extensions (solid rectangular frames). Potential antagonists are the extensor muscles during instructed flexions, the flexor muscles during instructcd extensions (dashed rectangular frames). But for a given muscle to serve as an agonist or antagonist of the instructed movement, that muscle must be able to act mechanically on the instructed digit or joint (e.g., wrist). Therefore, data from instructed movements during which a given muscle could act mechanically on the instructed digit or joint are shown as solid symbols; data from instructed movements during which the muscle could not act on the instructed digit or joint are shown as open symbols.

\section{Muscular production of instructed movements}

By examining the column of data for a given instructed movement in Figure 5 (or the row for a given movement in Fig. 4), the agonist, antagonist, and stabilizing activity in different muscles can be interpreted to form a tentative explanation of how the muscles produced each instructed movement. The motion
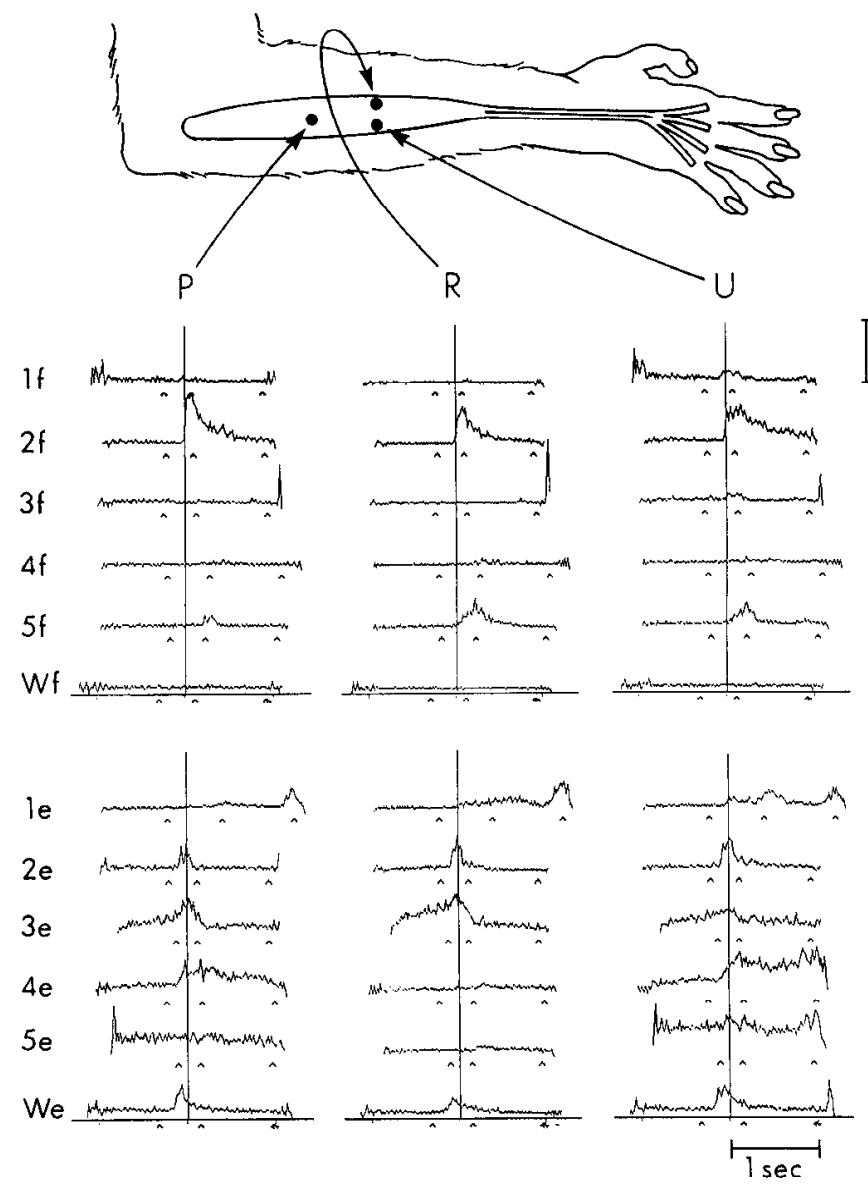

Figure 3. Simultaneous recordings from three locations in EDC. Three columns of histograms show EMG activity recorded simultaneously from electrodes placed proximally $(P)$ and more distally in the radial $(R)$ and ulnar $(C)$ aspects of EDC. The sketch above shows the approximate locations of the electrode insertions on a map of the extremity, relative to the presumed location of EDC. Electrodes $R$ and $U$ were separated by $9 \mathrm{~mm}$. Except for minor differences described in the text, all three electrodes recorded similar activity. Conventions for histograms are as for Figure 1. Vertical calibration, 750 arbitrary integrated EMG units.

observed in the instructed digit and in the noninstructed digits during each instructed movement has been described in detail previously (Schieber, 1991). Three examples are presented here to illustrate how the observed muscle activity could account for the observed finger movements.

If. During instructed thumb flexion, the thumb flexed with little if any motion of noninstructed digits (Schieber, 1991). Thumb flexion was produced by contraction of the thenar muscles. The only other muscle capable of flexing the thumb, FDPr, was silent. Little if any antagonist or stabilizing activity appeared in other muscles. Because the thenar muscles acted only on the thumb, and other muscles were inactive, noninstructed digits remained stationary.

$3 f$. During instructed flexion of the middle finger, digit 3 flexed the most, but was accompanied by somewhat less flexion of digits 2 and 4, and still less flexion of digit 5; digit 1 and the wrist remained stationary (Schieber, 1991). Movement $3 f$ involved EMG activity in FDPr, FDS, and FDPu, all acting as agonists of digit 3 flexion. Presumably, although each of these muscles inserts on digit 3 , none of them acting alone could flex 

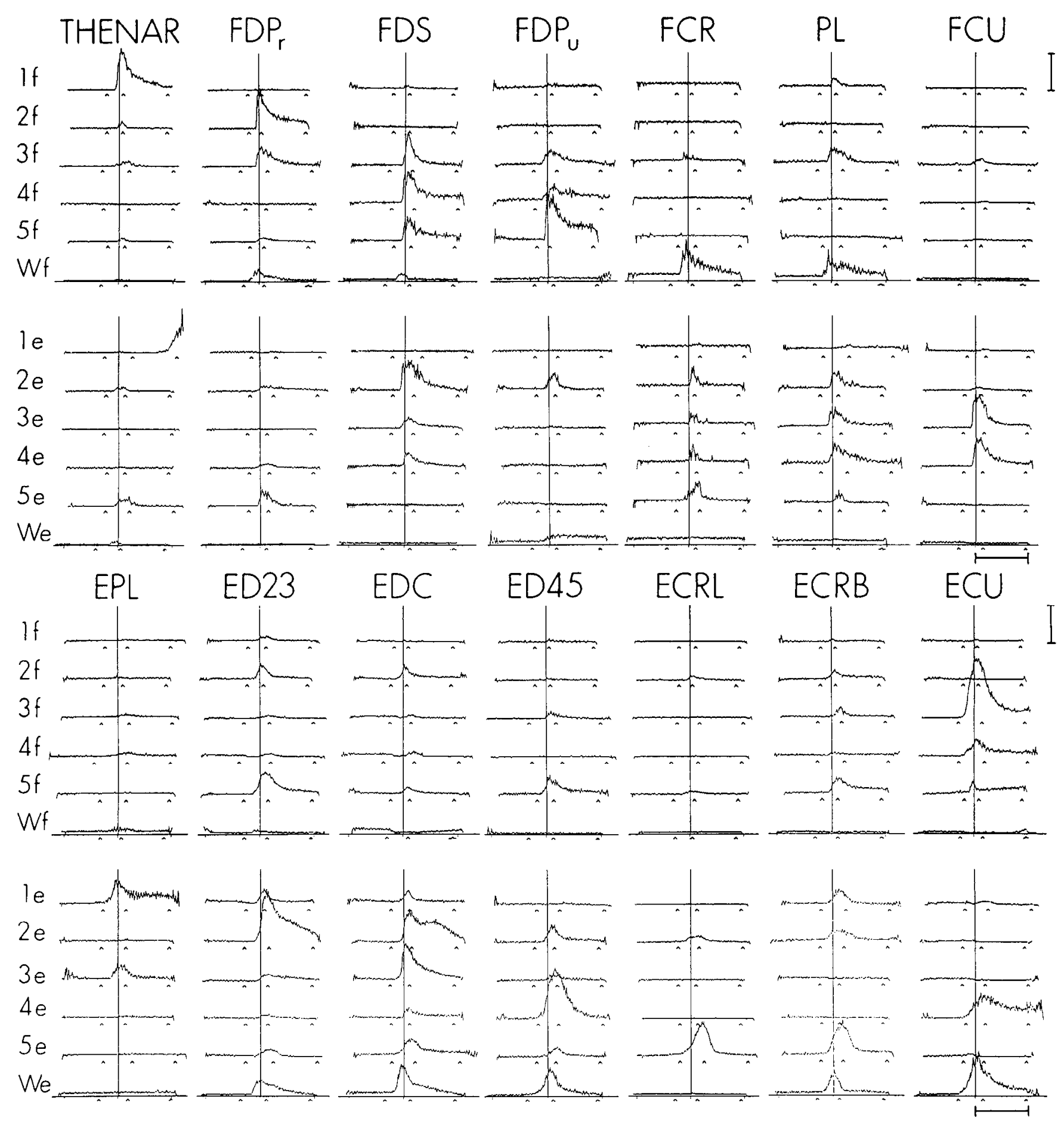

Figure 4. A typical EMG recording from each of the 14 muscles studied. All examples shown are from monkey $\mathrm{K}$. Conventions for histograms are as for Figure 1. Horizontal calibration, $1 \mathrm{sec}$; vertical calibration (arbitrary integrated EMG units): THENAR, 1000; FDPr, 1000; FDS, 500; $F D P u, 500 ; F C R, 250 ; P L, 500 ; F C U, 750 ; E P L, 250 ; E D 23,1000 ; E D C, 1000 ; E D 45,1000 ; E C R L, 750 ; E C R B, 1000 ; E C U, 1000$.

that digit in advance of the others; but when they acted together, the net tension on digit 3 exceeded that on other digits, flexing digit 3 more than the others. Because these muscles also acted on noninstructed digits 2,4 , and 5 , these digits also flexed somewhat. Little if any activity appeared in the potential antagonists, EDC or ED23. Contraction of FDPr, FDS, and FDPu would also have generated torque tending to flex the wrist. To stabilize the wrist by counterbalancing this wrist-flexing torque, ECU was intensely activated, with some cocontraction of PL. Con- traction of FDPr was insufficient to move the thumb, and other muscles acting on the thumb - thenar and EPL-were quiescent; hence, the thumb remained stationary.

$5 f$. During instructed flexion of the little finger, digit 5 flexed the most, but digit 4 also flexed considerably, and digit 3 flexed somewhat as well. Digit 2 typically extended. The thumb and wrist remained relatively stationary (Schieber, 1991). Movement $5 \mathrm{f}$ involved agonist activity in FDS and FDPu. The activity of FDS and FDPu also flexed noninstructed digits 3 and 
FLEXIONS

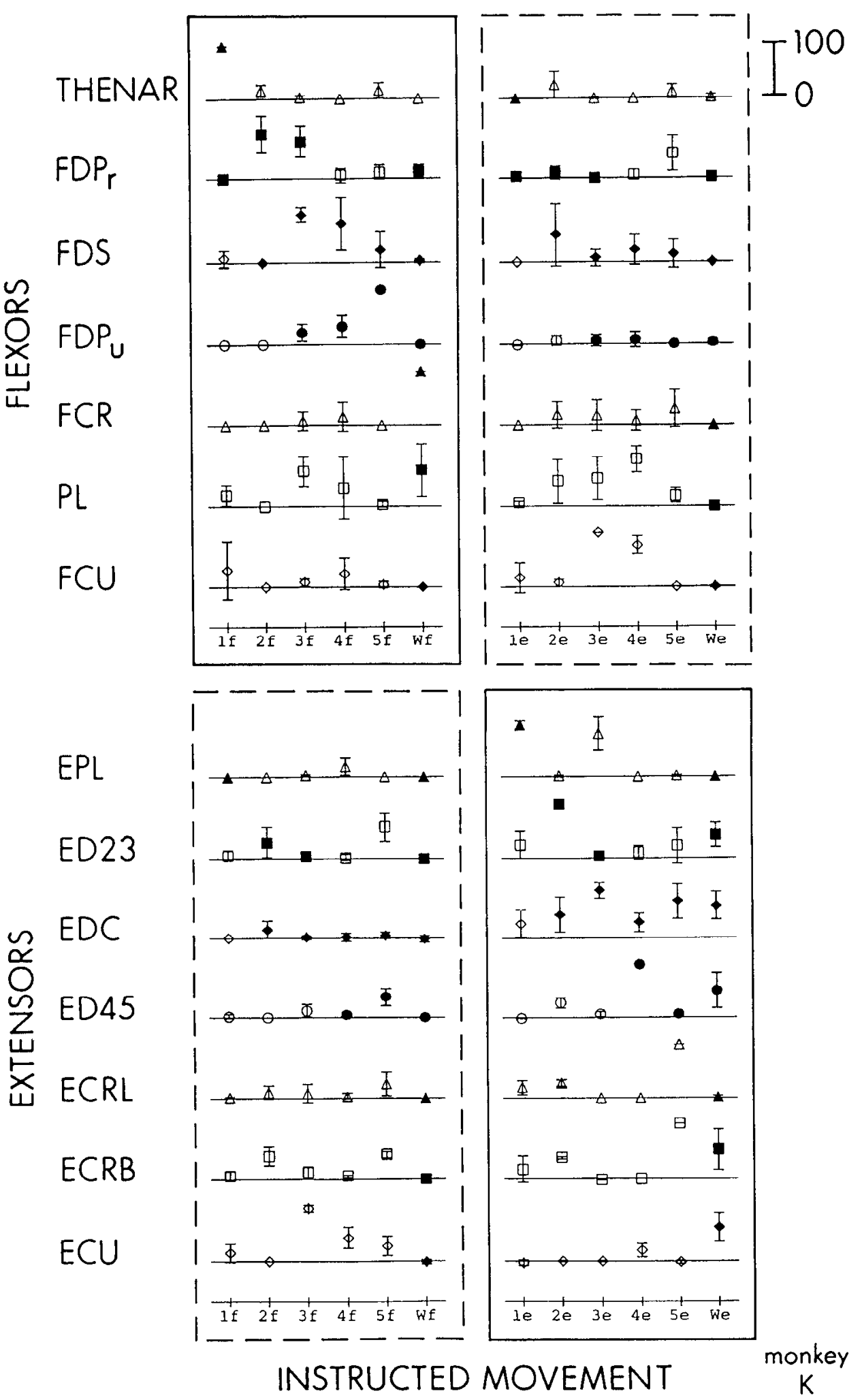

\section{EXTENSIONS}

Figure 5. Normalized (percentage maximum) changes in EMG activity for each instructed movement averaged across all recordings from each muscle in monkey $K$. Data are plotted as the mean \pm standard deviation. Different symbols (triangle, square, diamond, cir$c l e$ ) are used to distinguish the data from different muscles. Potential agonists (solid rectangular frames) are the flexors during instructed flexions (upper left) and the extensors during instructed extensions (lower right). Potential antagonists (dashed rectangular frames) are the flexors during instructed extensions (upper right) and the extensors during instructed flexions (lower left). However, each muscle can act as an agonist or antagonist of movement in the instructed digit only if its tendon can act mechanically on that digit (solid symbols). Instances in which a muscle that could not affect the instructed digit (open symbols) was active suggest a contraction that served to stabilize a noninstructed digit. Vertical scale bar (upper right) indicates $0-100 \%$ of the maximum change for each recording.
4. Some antagonist activity appeared in ED45 or EDC, which may also have served in part to stabilize digits 3 and 4 against flexing excessively with digit 5 . Stabilizing activity in ED23 may also have acted to prevent excessive flexion of digit 3 , while actually extending digit 2 . Stabilizing activity in the wrist extensors-ECRL, ECRB, and ECU - prevented flexion of the wrist that would otherwise have resulted from the wrist-flexing torques produced by FDS and FDPu. 


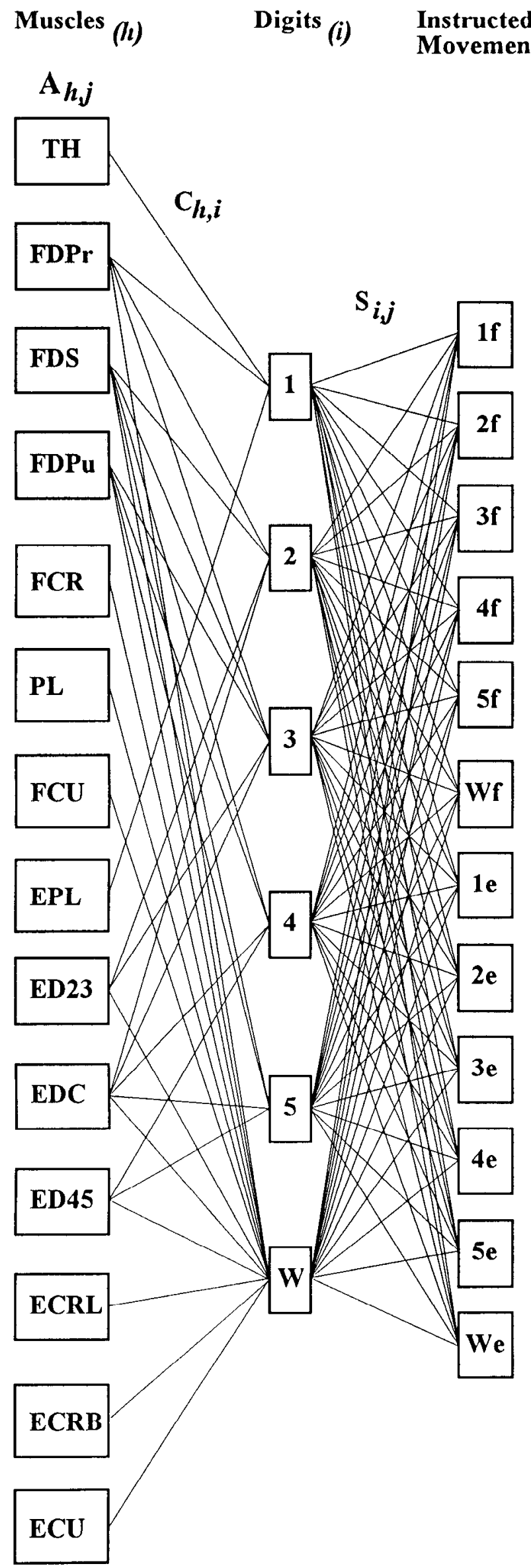

A model of the muscular production of finger movements

The plausibility of the observed patterns of EMG activity resulting in the present individuated finger movements via the agonist, antagonist, and stabilizing roles described above was explored further using a three-level connection model (Fig. 6). The model's output level consisted of the 12 instructed movements. These each received a contribution from each of the elements of the second level-the digits. The contribution of each digit to a given instructed movement was quantified as the slope of the relatively linear trajectory formed by plotting the position of that digit as a function of the position of the instructed digit during that instructed movement (see Figs. 4, 5 of Schieber, 1991). These slopes thus represent normalized position change of a given digit per normalized position change of the instructed digit, which is technically dimensionless. Such slopes were found empirically to be relatively (1) constant from Irial to trial, and (2) unaffected by variations in the initial and final positions of the noninstructed digits, which did vary considerably from trial to trial. Values for these slopes, averaged across all trials of each instructed movement in a session, were used as target values for the model to achieve. The values used for monkey $\mathrm{K}$ were those previously published (see Table 2 of Schieber, 1991). In this previous work, slopes had been assigned positive values if a digit moved in the same direction as the instructed digit, and negative values if it moved in the opposite direction. For purposes of the present modeling, the sign of the slopes during instructed extensions was changed, so that for all instructed movements all slopes had a positive value if the digit was flexing and a negative value if the digit was extending. This alteration permitted the slope of each digit to be computed on a continuous scale ranging from approximately +1.0 (digit flexed fully), through 0.0 (digit did not move), to -1.0 (digit extended fully).

The digits of the model's second level each received input from certain elements of the third level-muscles. In the model, each muscle was allowed to affect only those digits upon which it can act mechanically in vivo. A muscle's contribution to the slope of a digit during a particular instructed movement was computed as that muscle's EMG activity change during the movement, multiplied by a weighting constant specific for that muscle-digit coupling. Data like those displayed in Figure 5 were used as the EMG activity changes. The slope of a given digit during a particular instructed movement then was modeled as the sum of contributions from all muscles:

\footnotetext{
$\leftarrow$

Figure 6. Model of the production of individuated finger movements by EMG activity changes. The model used three levels. The output level consisted of the 12 instructed movements. These each received a contribution from each of the elements of the second level-the digits. The contribution of each digit $(i)$ to a given instructed movement $(j)$ was quantified as the slope, $S_{t, j}$, which expresses the motion of the that digit relative to the instructed digit during the given instructed movement (Schicber, 1991). The digits of the model's second level each received input from certain elements of the third level-muscles. In the model, each muscle was allowed to affect only those digits upon which it can act mechanically in vivo. A muscle's $(h)$ contribution to the slope of a particular digit during a given instructed movement then was computed as that muscle's EMG activity change during the movement, $A_{h, j}$, multiplied by a weighting constant specific for that muscle-digit coupling, $C_{h, i}$. The constants, $C_{h, i}$, which were the same for all 12 instructed movements, were iteratively adjusted to optimize the fit between the slopes, $S_{i, j}$, computed by the model and target values derived from experimental
} data. 


$$
S_{i, j}=\sum_{h=1}^{q} C_{h, i} \cdot A_{h, j}
$$

where $S_{i, j}$ is the slope of the $i$ th digit during the $j$ th instructed movement, $C_{h, i}$ is the constant coupling EMG activity changes in the $h$ th muscle to the slope of the $i$ th digit for all movements, $A_{h, j}$ is the activity change of the $h$ th muscle during the $j$ th instructed movement, and $q$ is the number of muscles under consideration (here $q=14$ ).

The constants, $C_{h, i}$, which were the same for all 12 instructed movements, were iteratively adjusted with a gradient descent algorithm to optimize the fit between slopes computed by the model and slopes derived from experimental data. Constants for flexor muscles were given positive values since these muscles acted to flex the digits (positive slope); constants for extensor muscles were given negative values since these muscles acted to extend the digits (negative slopes). Each constant was initially set to a best guess value. Then, in a fixed rotation, each constant was randomly increased or decreased by $10 \%$, and the error (the absolute value of the modeled slope minus the target slope, with slope dimensions as above) was totalled for all six digits during all 12 instructed movements. (E.g., if the slope calculated by the model for each digit during each instructed movement had differed from the observed slope by 1.0 , the total error would have been 72.0.) If the altered constant reduced the total error, the alteration was retained; if not, the constant was reset to its previous value.

When EMG activity alone was used as input to the model, the finger slopes calculated during instructed movement We indicated that the fingers extended, when in fact they remained stationary. Since the finger flexors are known to have a passive elastic element that acts to flex the fingers as the wrist extends (cf. Long et al., 1966), such a passive elastic element was incorporated in the model (not illustrated in Fig. 6). After each iteration calculating the slopes by the formula above, the calculated wrist slope, if negative (indicating extension), was multiplied by a separate positive constant representing the passive elastic effect of the finger flexors on digit $2,3,4$, or 5 , and the resulting terms were subtracted from the slopes calculated for digits $2-5$, respectively. Total error of the fit was calculated in each iteration after adding these passive elastic terms. Incorporating these elastic terms improved the fit of digits $2-5$ during We.

Figure 7 shows the best fit achieved by the model using the EMG activity changes shown in Figure 5 to fit target slopes previously derived from experimental data for monkey $\mathrm{K}$ (see Table 2 of Schieber, 1991). Table 2 gives the constants, $C_{h, i}$, produced by the model in achieving this fit. The model accounted for most of the motion of both the instructed and noninstructed digits in each instructed movement. In movement $5 \mathrm{f}$, for example, the model accounted reasonably well not only for the full range flexion of digit 5 , but also for the lesser flexion of digits 4 and 3 , for the lack of motion of digit 1 or the wrist, and even for the extension of digit 2 . The total error for the fit shown in Figure 7 was 8.1. Slopes and EMG activity changes from monkey $\mathrm{S}$ or from monkey $\mathrm{H}$ were also used separately as target values and input for the model. Since no data were available from EPL or ED23 in monkey S, or from ED23 in monkey $\mathrm{H}$, data from these muscles in monkey $\mathrm{K}$ were substituted. The best fit achieved for monkey $\mathrm{S}$ had a total error of 11.4 , and the best fit for monkey $\mathrm{H}$ had a total error of 11.7 . The fits achieved by the model support the notion that each
Table 2. Optimized EMG-to-slope coupling constants, $C_{h, i}\left(\times 10^{3}\right)$

\begin{tabular}{|c|c|c|c|c|c|c|}
\hline \multirow{2}{*}{$\begin{array}{l}\text { Muscle } \\
(h)\end{array}$} & \multicolumn{5}{|c|}{ Digit $(i)$} & \multirow[b]{2}{*}{ W } \\
\hline & 1 & 2 & 3 & 4 & 5 & \\
\hline Thenar & 10.0 & & & & & \\
\hline FDPr & 0.5 & 10.9 & 8.2 & & & 2.8 \\
\hline FDS & & 0.7 & 4.8 & 10.7 & 0.7 & 4.0 \\
\hline $\mathrm{FDPu}$ & & & 4.3 & 7.2 & 12.8 & 2.7 \\
\hline FCR & & & & & & 8.5 \\
\hline PL & & & & & & 1.8 \\
\hline $\mathrm{FCU}$ & & & & & & 0.3 \\
\hline EPL & -8.5 & & & & & -0.1 \\
\hline ED23 & & -9.0 & -3.1 & & & -0.6 \\
\hline EDC & & -5.5 & -9.1 & -4.8 & -6.7 & -3.2 \\
\hline ED45 & & & & -11.0 & -5.5 & -0.8 \\
\hline ECRL & & & & & & -1.1 \\
\hline ECRB & & & & & & -1.5 \\
\hline $\mathrm{ECU}$ & & & & & & -7.2 \\
\hline Elastic & & 8.9 & 8.1 & 7.3 & 6.0 & \\
\hline
\end{tabular}

Values represent the coupling constants, $C_{h, i}$, multiplied by $10^{3}$ to remove leading zcros. The order of magnitude of the constants reflected that they converted EMG activity, normalized in the $0-100 \%$ range, to slopes in the -1 to +1 range. The constants for flexor muscles are positive since their activity acted to fiex digits (positive slope); the constants for extensor muscles are negative since their activity acted to extend digits (negative slope). Each empty table cell indicates that the model used no connection between that muscle and that digit. Constants in the bottom row, "Elastic," represent the passive elasticity of the finger flexor muscles, which in the model acted to flex digits $2-5$ in proportion to the wrist extension.

individuated finger movement was produced by different levels of activity in a number of monotendoned and multitendoned muscles-some acting as agonists, some as antagonists, and some as stabilizers-combined such that the net effect was movement of the instructed digit more than others.

Although the model accounted for most of the movement of both the instructed and noninstructed digits, a number of discrepancies were evident. Most notable perhaps was the slope computed for digit 5 during instructed movement $2 \mathrm{e}$. The slope computed by the model suggested that digit 5 extended, whereas during $2 \mathrm{e}$, digit 5 actually flexed. This discrepancy suggests that some muscle contraction(s) not recorded in the present study occurred during 2e to flex digit 5 . Similar discrepancies are reflected in the less accurate fits (with higher total error) achieved for monkeys $\mathrm{S}$ and $\mathrm{H}$. In each of these monkeys, the model failed to fit flexion of the thumb during movement $1 \mathrm{f}$. In monkey $\mathrm{S}$, the thenar muscles were active during movements $2 \mathrm{e}, 4 \mathrm{e}$, and $5 \mathrm{e}$, when they were unopposed by activity in EPL (data substituted from monkey $\mathrm{K}$ ). If the model used a substantial coupling of thenar EMG activity to the thumb, then the thumb was calculated to flex during all these movements as well, contributing approximately 3 units of error. But if the model uncoupled thenar EMG activity from thumb movement by lowering the coupling constant, then the calculated thumb motion only failed to flex during movement 1 f, contributing only 1 error unit. In monkey $\mathrm{H}$, thenar EMG activity increased with movements le, $2 \mathrm{e}, 3 \mathrm{e}$, and $\mathrm{We}$, and tonic activity preceding movements $2 \mathrm{f}$ and 4f decreased, with EPL cocontracting only during le. These thenar EMG activity changes again caused the model to minimize the constant coupling thenar EMG to thumb flexion. As with the discrepancy between the observed and calculated motion of digit 5 during $2 \mathrm{e}$ in monkey $\mathrm{K}$, these discrepancies for thumb flexion in monkeys $\mathrm{S}$ and $\mathrm{H}$ suggest that unobserved 


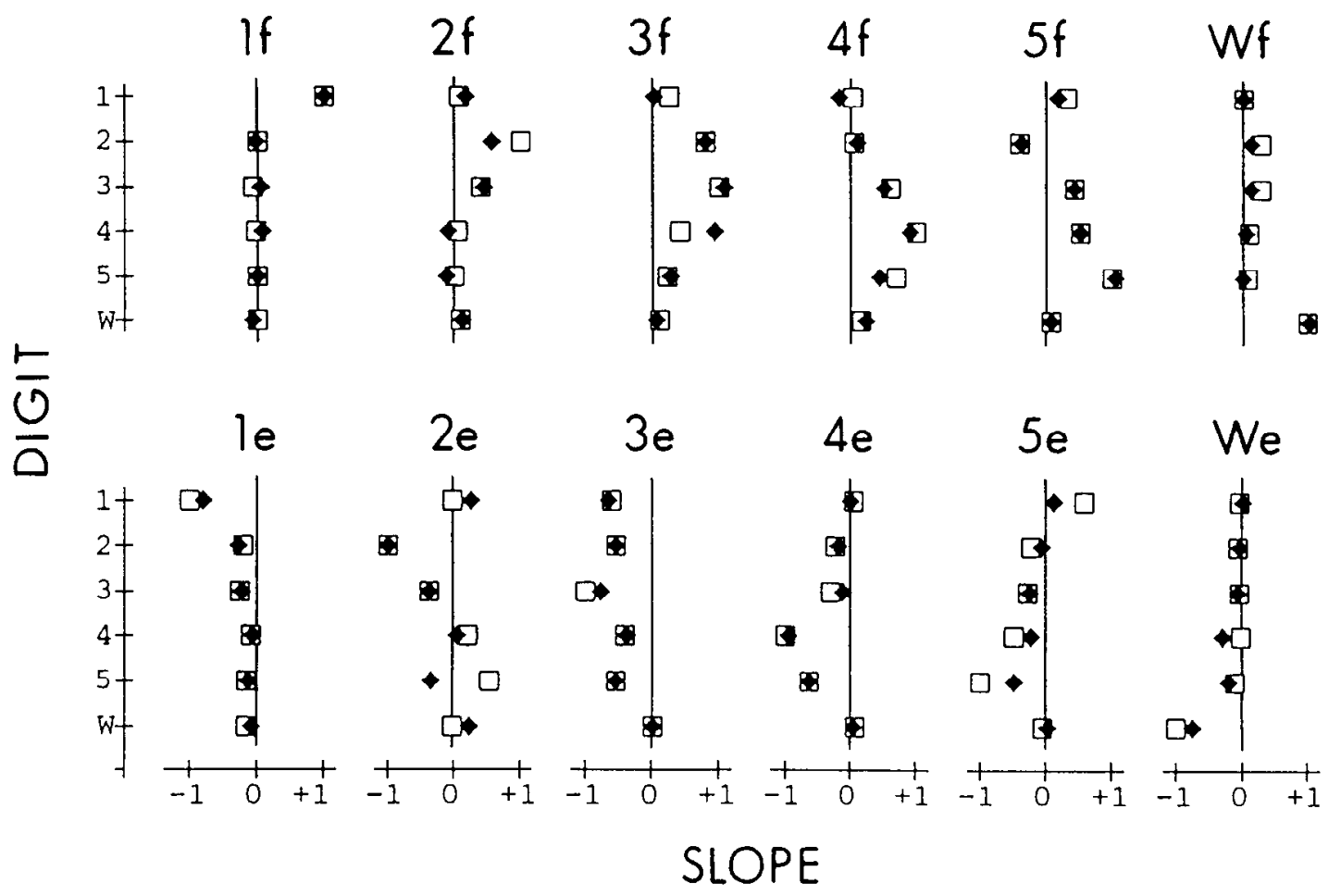

Figure 7. Fit of slopes $\left(S_{i, j}\right)$ computed by the model from EMG activity changes $(\downarrow)$ to slopes derived from experimental data ( $\square$ ) for each digit (i) during each instructed movement $(j)$.

muscle contractions affected the thumb. Finally, in both monkcys $\mathrm{S}$ and $\mathrm{H}$, the model failed to calculate extension of the wrist during instructed movement We. Similar to the discrepancy in thumb flexion, this appeared to result from substantial activity of each wrist extensor during several instructed movements other than We, that the model was unable to counterbalance with other activity.

\section{Discussion}

The present study shows that individuated finger movements performed by rhesus monkeys typically involved simultaneous EMG activity in several muscles, some acting as an agonist of the instructed movement, some acting as an antagonist, and others acting to stabilize noninstructed digits. EMG activity changes in agonists, antagonists, and stabilizers also accounted for motion of noninstructed digits, in part because extrinsic finger muscles functioned as multitendoned muscles. Although recordings showed complex combinations of various degrecs of EMG activity changes in different muscles during different movements, a model that computed the relative motion (slopes) of the digits during each instructed movement demonstrated that these EMG activity changes could have produced the observed movements. The simultaneous movement of the instructed and noninstructed digits thus was accounted for by the net effects of several muscles acting simultaneously on many or all digits.

\section{Describing the functional roles of the extrinsic finger muscles}

The traditional terms "agonist" and "antagonist" are most applicable when considering two oppositely directed movements about a joint. In such a situation, the number of available muscles often exceeds the number of studied movements. A group of "synergists" may then be coactivated in each movement.
Simple wrist flexion and extension exemplify such a situation. The wrist flexors per se, as well as the extrinsic finger flexors, may be coactivated as synergists, serving as agonists for flexion and antagonists for extension; conversely, the wrist extensors and extrinsic finger extensors may be coactivated as agonists for extension and antagonists for flexion (Cheney and Fetz, 1980; Fetz and Cheney, 1980; Schieber and Thach, 1985).

As the number of studied movements approaches, equals, and then exceeds the number of available muscles, however, different movements must be achieved by contracting the available muscles to different degrees. By combining various lcvels of activity in participating muscles, more distinct movements can be produced than there are muscles to produce them. Describing a muscle as an agonist or antagonist of a particular movement then becomes inadequate. Such a situation has been studied by Hoffman and Strick (1986), who examined the muscle activity used to achieve 12 different combinations of wrist flexion/extension and radial/ulnar deviation. In this task, ECRL was active during movements in several of the 12 directions, showing quantitatively different degrees of activity for wrist movements in different directions. Describing ECRL as an agonist of wrist extension would fail to convey (1) that the same muscle was active for movements in many other directions as well, and (2) that quantitatively different levels of activity in the same muscle contributed to production of different movements.

Similarly in the present individuated finger movements, the number of movements approached the number of muscles involved, and the movements were produced by different combinations of various degrees of EMG activity in the available muscles. A given muscle contributed to several distinct finger movements with quantitatively different degrees of EMG activity change. Though FDPu, for example, could be described as 
an agonist of instructed movements $3 f$, $4 f$, and $5 f$, a critical factor in producing these three distinct movements was that FDPu was slightly active during $3 f$, more active during $4 \mathrm{f}$, and most active during $5 \mathrm{f}$. A second critical factor was that a multitendoned muscle like FDPu produces mechanical actions on multiple digits whenever it contracts. Such a muscle cannot be described as an agonist, antagonist, or stabilizer of a given digit. Prcsumably FDPu, whenever it was active, produced the same proportional distribution of tension across digits 3,4 , and 5 , scaled in amplitude according to the level of EMG activity during $3 f, 4 f, 5 f$, or any other movement. Which movement occurred depended, then, on how the various levels of activity in FDPu were combined with different levels of activity in FDPr, in FDS, and in other muscles.

\section{Sources of intra- and interindividual variability in recorded EMG activity}

As long as fewer movements are examined than there are muscle combinations available to produce them, different muscle combinations might be used by different subjects, or by the same subject in different sessions, to produce a given movement (Loeb, 1993). During instructed movement $5 \mathrm{f}$, for example, stabilization of the wrist against the flexing torque resulting from activity in FDPu and FDS was provided by various combinations of wrist extensor activity in the three monkeys. Monkey $\mathrm{K}$ activated ECRB relatively more than ECRL or ECU; monkey $S$ activated only ECU; monkey $H$ activated ECU relatively more than ECRL or ECRB. Although flexion/extension motion of the wrist had to be controlled actively by the monkey, radial/ulnar deviation of the wrist was limited passively by the manipulandum, and was not otherwise monitored. Each monkey therefore was free to acquire an idiosyncratic use of muscle activity that might otherwise have affected radial/ulnar stabilization of the wrist. Had the monkeys been required to control actively the wrist's radial/ulnar degree of freedom, and to produce distinct radial/ulnar wrist movements (e.g., digit 5 flexion with wrist radial deviation, and digit 5 flexion with wrist ulnar deviation), more consistent patterns of activation in ECRL, ECRB, and ECU might have been observed. Besides the 14 muscles monitored in the present study, unmonitored muscles-intrinsic hand muscles or unidentified functional subdivisions of the extrinsic finger muscles-may have made additional muscle combinations available. The availability of more than one muscle combination capable of producing the features required for a given instructed movement thus may account for some of the variability observed among monkeys, or across sessions in a given monkey (error bars in Fig. 5).

A second potential source of variability in recorded EMG activity may have been variation in the placement of recording electrodes. If a muscle had differentially activated subdivisions that were not identified in the present study, then the pattern of EMG activity recorded from the muscle would have varied with the exact location of electrodes in the muscle. This may account for some of the variation among the three simultaneous recordings from EDC shown in Figure 3. Similarly, EMG activity picked up from adjacent muscles (despite the present efforts to eliminate such pickup and to exclude contaminated recordings) would have varied with electrode position in the impaled muscle, and could potentially have caused similar variability in the recorded EMG.

Third, recent studies of the cat hindlimb have shown that a given muscle may have significant interindividual variation in biomechanical parameters, such as the moment arm of the tendon about a joint (Young et al., 1993). Though biomechanical parameters of the muscles were not measured in the present study, the constants optimized by the model suggest some differences among monkeys. For example, the model's best fits used the largest constants representing passive elasticity of the finger flexors during wrist extension for monkey $\mathrm{K}$ (the elastic constant for digit 2 was 8.9 for monkey $\mathrm{K}, 1.4$ for monkey $\mathrm{S}$, and 2.7 for monkey $H$ ), consistent with monkey $K$ having a larger forearm flexor muscle mass, presumably with greater elasticity than the other monkeys. Such biomechanical differences among individual monkeys, which could be measured directly in other experiments, thus may be another source of variability in the present study.

\section{Modeling the muscular production of finger movements}

The net effect of all muscles active in each movement was summarized most succinctly by the model, which used each muscle's EMG activity change during each movement to compute the relative motion slope of each digit. This model was able to account for most of the motion of both the instructed and the noninstructed digits. To do so, the EMG-to-slope constants for each muscle-digit coupling were optimized with a gradient descent algorithm. The conversion of EMG activity in a muscle to movement of a digit involves several intermediate steps, however, which are not necessarily linear functions of EMG activity. These steps include (1) the conversion from EMG amplitude to tension generated in the tendon, which probably is relatively linear (Lippold, 1952; Bigland and Lippold, 1954; Schieber and Thach, 1985); (2) the conversion from tension in the tendon to torque gencrated at the joints, which may involve different moment arms for different tendons, and variation in the moment arm of a single tendon during movement (Young et al., 1993); (3) the conversion from joint torques to resultant joint motion; and, (4) for multitendoned muscles, mechanical interactions between the tendons to different digits. The present analysis suggests that the empirically derived EMG-to-slope coupling constants represent all these factors lumped together, but available data are inadequate to test this hypothesis directly, which would require experimental measurement of each factor.

Although the experimental data needed to derive the EMGto-slope constants are not available for any of the muscle-digit couplings studied here, the constants optimized by the model may be reasonable empiric estimates. In particular, the constants for FDPr suggest that a given amount of EMG activity in FDPr would produce the most flexion in digit 2, some flexion in digit 3, and little if any flexion in digit 1 ; similarly, the constants for FDPu suggest that a given amount of EMG activity in $\mathrm{FDPu}$ would produce the most flexion in digit 5, somewhat less in digit 4 , and the least flexion in digit 3 . The order of these effects on different digits parallels previously reported effects of intramuscular stimulation of FDPr or FDPu: stimulation of FDPr produced greatest flexion of digit 2, less of digit 3 , and nil of digit 1; stimulation of FDPu produced greatest flexion of digit 5, somewhat less of digit 4, and still less of digit 3 (Schieber, 1993a). If a similar parallelism holds for the relative effects of each multitendoned muscle on its different tendons, then the optimized constants in Table 2 also predict the following: contraction of FDS should flex digit 4 more than digit 3, contraction of EDC should extend digit 3 more than other digits, contraction of ED23 should extend digit 2 more than 3, and contraction of ED45 should extend digit 4 more than 5 . These predictions 
could be tested in other experiments by directly measuring the tension in each tendon of a given muscle resulting from a given level of either EMG activity or electrical stimulation (cf. Fritz et al., 1992).

Although the model accounted for most of the motion of both the instructed and noninstructed digits, a number of discrepancies were evident between the observed and calculated motion. In part, such discrepancies may have resulted from the use of EMG activity taken from many recording sessions to fit finger movements averaged from only a single session, but these discrepancies may also suggest that some muscle activity not identified in the present study sometimes affected the motion of certain digits. Three possible sources of such unidentified EMG activity can be considered. First, many muscles controlling the fingers were not studied here. Intrinsic hand muscles, such as flexor digiti quinti brevis, contrahentes digiti quinti, or the seventh palmar interosseus muscle, may have flexed digit 5 during $2 \mathrm{e}$ in monkey $\mathrm{K}$, and contributed to flexion of digit 5 during other instructed movements as well (Howell and Straus, 1933). The extrinsic muscle, abductor pollicis longus, may have helped extend the thumb and oppose thenar muscle activity in monkeys $\mathrm{S}$ and $\mathbf{H}$. Second, the multitendoned extrinsic finger muscles may have functional subdivisions from which recordings were not obtained in the present study. FDS's tendon to digit 5, for example, receives muscle fibers from only a small region of the muscle (FDSdu5; Serlin and Schieber, 1993). The techniques used to place EMG electrodes for the present study may have positioned electrodes in this small region only sporadically, failing to identify it consistently as a functional subdivision of FDS. Third, some discrepancies may have resulted from attributing recorded EMG activity to the wrong muscle. For example, all recordings from thenar muscles in the present study were pooled together. This pooling would have caused no discrepancy as long as the thumb flexors (e.g., flexor pollicis brevis) were active whenever other thenar muscles were active. But if abductor pollicis brevis had been active without the thumb flexors, EMG activity would have been recorded without production of torque flexing the thumb. Such inaccurate attribution of thenar EMG activity during movements other than $1 \mathrm{f}$ would have caused the model to uncouple thenar EMG activity from thumb flexion. Further studies of the intrinsic hand muscles, and of possible functional subdivisions in the multitendoned extrinsic finger muscles, will be needed for a more complete description of the muscular production of individuated finger movements.

Two simplifications used here to achieve a tractable model should be noted. First, the present model used only changes in EMG activity to compute relative changes in the positions of the digits. Eventually, tonic levels of EMG activity should be incorporated to account for the maintained positions of the fingers. Second, the present model did not incorporate the time course of either EMG activity changes or movements. Eventually, these time courses should be included for a more complete description of individuated finger movements.

\section{Muscular limitations on the ability to individuate finger movements}

The present study assumes that the macaque multitendoned extrinsic finger muscles do not operate as separate functional subdivisions for each digit. Consistent evidence of functional subdivisions has been found only for FDP, where two major regions act on different subsets of tendons to the five digits: FDPr on tendons to digits 1, 2, and 3; and FDPu on tendons to digits 3, 4, and 5 (Schieber, 1993a; Serlin and Schieber, 1993). EMG evidence of functional subdivisions in other multitendoned extrinsic finger muscles was not observed during the present finger movements. Conceivably, had more complete individuation of the present finger movements, or more different movements, been required of the present monkeys, functional subdivisions that were coactivated here might have been differentially activated. However, given the extensive training of the present monkeys (12-24 months) and the degree of difficulty they encountered with the task, they can reasonably be assumed to have performed to their maximum potential. Moreover, recordings of single motor units in multitendoned muscles typically showed that a given motor unit discharged during more than one instructed movement for which the parent muscle showed agonist EMG activity (cf. Schieber, 1993a). Concluding tentatively then that each multitendoned muscle, or functional subdivision thereof (e.g., FDPr and FDPu), applies tension to more than one digit at a time, it would follow that macaque finger movements could not be completely independent. Even if the tendons from each of these muscles to the digits it serves is mechanically independent, the muscles could not operate the digits independently without a separate functional subdivision of the muscle belly for each tendon.

The ability to individuate finger movements increases along the phylogenetic scale of mammals. Rats open and close their fingers in grasping food, cats flex and adduct their digits to grasp food morsels, monkeys pinch small objects between the tip of the thumb and the side of the index, humans finger musical instruments (Napier, 1960; Gorska and Sybirska, 1980; Wishaw et al., 1993). Though commonly attributed to phylogenetic enlargement of the corticospinal pathway in the nervous system, this increasing ability to individuate finger movements also parallels species differences in the structure of muscles (Serlin and Schieber, 1993). For example, whereas the macaque FDP has a sheet-like tendon that divides to all five digits, the human FDP has separate tendons to each finger, and a separate muscle belly with its tendon-flexor pollicis longus-serves the thumb (Fahrer, 1981). The macaque ED23 and ED45 are replaced in humans by extensor indicus proprius and extensor digiti quinti proprius, respectively. Certain multitendoned muscles to digits 1,2 , and 5 in the macaque thus are superseded by monotendoned muscles in the human hand. Associated with these monotendoned muscles is the greater degree of independence commonly ascribed to human digits 1,2 , and 5 . Similarly, instructed movements if and Wf, which were produced here by monotendoned muscles, had the highest degree of individuation (Schieber, 1991). Although the lesser independence of digits 3 and 4 is often attributed to tendinous interconnections (Fahrer, 1981 ), in humans as in monkeys the muscles moving digits 3 and 4-FDS, FDP, and EDC-may not be able to act independently on their tendons to different fingers (S. C. Gandevia, personal communication). Human individuated finger movements, then, may also result from the net effect of several muscles - some monotendoned and some multitendoned - acting simultaneously on multiple digits.

\section{References}

Armstrong JB, Rose PK, Vanner S, Bakker GJ, Richmond FJR (1988) Compartmentalization of motor units in the cat neck muscle, biventer cervicis. J Neurophysiol 60:30-45.

Basmajian JV (1978) Muscles alive. Their functions revealed by electromyography, 4th ed. Baltimore, MD: Williams \& Wilkins.

Berringer OM, Browning FM, Schroeder CM (1968) An atlas and 
dissection manual of rhesus monkey anatomy. Tallahassee, FL: Anatomy Laboratory Aids.

Bigland B, Lippold OCJ (1954) The relation between force, velocity and integrated electrical activity in human muscles. J Physiol (Lond) 123:214-224.

Brandell BR (1970) An electromyographic-cinematographic study of the muscles of the index finger. Arch Phys Med Rehab 51:278-285.

Chanaud CM, Pratt CA, Loeb GE (1991) Functionally complex muscles of the cat hindlimb. V. The roles of histochemical fiber-type regionalization and mechanical heterogeneity in differential muscle activation. Exp Brain Res 85:300-313.

Cheney PD, Fetz EE (1980) Functional classes of primate corticomotoneuronal cells and their relation to active force. J Neurophysiol 44:773-791.

Cheney PD, Fetz EE, Palmer S (1985) Patterns of facilitation and suppression of antagonist forelimb muscles from motor cortex sites in the awake monkey. J Neurophysiol 53:805-820.

Close RJ, Kidd CC (1969) The functions of the muscles of the thumb the index, and long fingers. J Bone Joint Surg 51A:1601-1620.

Emonet-Dénand F, Laporte Y, Proske U (1971) Contraction of muscle fibers in two adjacent muscles innervated by branches of the same motor axon. J Neurophysiol 34:132-138.

English AW (1984) An electromyographic analysis of compartments in cat lateral gastrocnemius muscle during unrestrained locomotion. J Neurophysiol 52:114-125.

English AW, Weeks OI (1987) An anatomical and functional analysis of cat biceps femoris and semitendinosus muscles. J Morphol 191: 161-175.

English AW, Weeks OI (1989) Electromyographic cross-talk within a compartmentalized muscle of the cat. J Physiol (Lond) 416:327-336.

Fahrer M (1981) Interdependent and independent actions of the fingers. In: The hand (Tubania R, ed), pp 399-403. Philadelphia: Saunders.

Fetz EE, Cheney PD (1980) Postspike facilitation of forelimb muscle activity by primate corticomotoneuronal cells. J Neurophysiol 44: $751-772$

Fritz N, Schmidt C, Yamaguchi T (1992) Biomechanical organization of single motor units in two multi-tendoned muscles of the cat distal forelimb. Exp Brain Res 88:411-421.

Gorska T, Sybirska E (1980) Effects of pyramidal lesions on forelimb movements in the cat. Acta Neurobiol Exp 40:843-859.

Hammond CGM, Gordon DC, Fisher JT, Richmond FJR (1989) Motor unit territories supplied by primary branches of the phrenic nerve. J Appl Physiol 66:61-71.

Hoffman DS, Strick PL (1986) Activity of wrist muscles during steptracking movements in different directions. Brain Res 367:287-291.

Howell BA, Straus WL Jr (1933) The muscular system. In: Anatomy of the rhesus monkey (Hartman CG, Straus WL Jr, eds), pp 89-175. New York: Hafner.

Landsmeer JMF, Long C (1965) The mechanism of finger control, based on electromyograms and location analysis. Acta Anat 60:330 347.

Lemon RN, Mantel GWH, Rea PA (1990) Recording and identification of single motor units in the free-to-move primate hand. Exp Brain Res 81:95-106.

Lippold OCJ (1952) The relation between integrated action potentials in a human muscle and its isometric tension. J Physiol (Lond) 117: 492-499.

Loeb GE (1989) The functional organization of muscles, motor units, and tasks. In: The segmental motor system (Binder MD, Mendell LM, eds), pp 23-35. London: Oxford UP.

Loeb GE (1993) The distal hindlimb musculature of the cat: interanimal variability of locomotor and cutaneous reflexes. Exp Brain Res 96:125-140.

Loeb GE, Gans C (1986) Electromyography for experimentalists. Chicago: University of Chicago.

Long C (1968) Intrinsic-extrinsic muscle control of the fingers. J Bone Joint Surg 50A:973-984.

Long C, Brown ME (1964) Electromyographic kinesiology of the hand: muscles moving the long finger. J Bone Joint Surg 46:1683-1706.

Long C, Thomas D, Crochetiere WJ (1966) Viscoelastic factors in hand control. In: International congress series, No 107, Proceedings of the fourth international congress on physical medicine, Paris 1964 , pp 440-451. Excerpts Medica.

Long C, Conrad PW, Hall EA, Furler SL (1970) Intrinsic-extrinsic muscle control of the hand in power grip and precision handling. $J$ Bone Joint Surg 52A:853-867.

Napier JR (1960) Studies of the hands of living primates. Proc Zool Soc Lond 134:647-657.

Schieber MH (1991) Individuated finger movements of rhesus monkeys: a means of quantifying the independence of the digits. J Neurophysiol 65:1381-1391.

Schieber MH (1993a) Flectromyographic evidence of two functional subdivisions in the rhesus monkey's flexor digitorum profundus. Exp Brain Res 95:251-260.

Schieber MH (1993b) Muscular production of individuated finger movements. Soc Neurosci Abstr 19:548.

Schieber MH, Thach WT Jr (1985) Trained slow tracking: I. Muscular production of wrist movement. J Neurophysiol 54:1213-1227.

Serlin DM, Schieber MH (1993) Morphologic regions of the multitendoned extrinsic finger muscles in the monkey forearm. Acta Anat 146:255-266.

Szebenyi ES (1969) Atlas of Macaca mulatta. Rutherford, NJ: Farleigh Dickinson UP.

Wishaw IQ, Pellis SM, Boguslaw G, Kolb B, Wolfram T (1993) Proximal and distal impairments in rat forelimb use in reaching follow unilateral pyramidal tract lesions. Behav Brain Res 56:59-76.

Young RP, Scott SH, Loeb GE (1993) The distal hindlimb musculature of the cat: multiaxis moment arms at the ankle joint. Exp Brain Res 96:141-151. 